\title{
Molecular differentiation and pathogenicity of Aviadenoviruses isolated during an outbreak of inclusion body hepatitis in South Africa
}

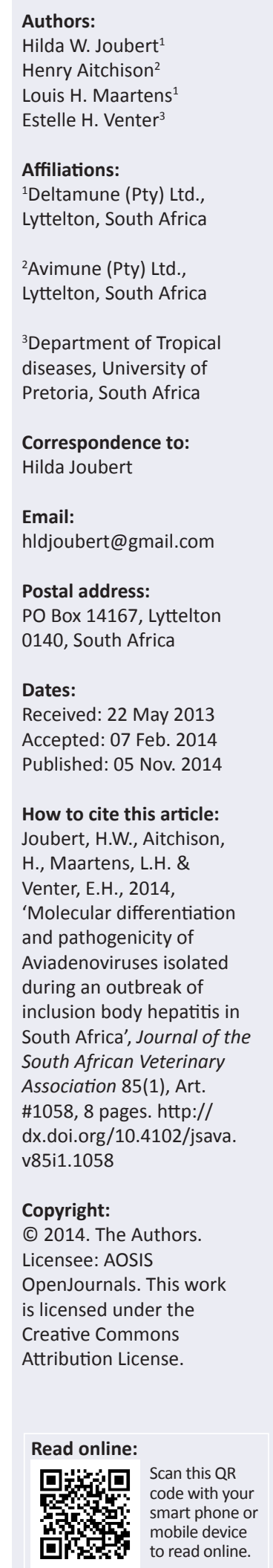

Fowl adenovirus (FAdV) is a member of the genus Aviadenovirus and causes a number of economically important poultry diseases. One of these diseases, inclusion body hepatitis (IBH), has a worldwide distribution and is characterised by acute mortality $(5 \%-20 \%)$ in production chickens. The disease was first described in the United States of America in 1963 and has also been reported in Canada, the United Kingdom, Australia, France and Ireland, but until now, not in South Africa. Adenoviruses isolated from the first outbreak of IBH in South Africa were able to reproduce the disease in chicken embryo livers. The aim of the present study was to characterise the viruses and determine the pathogenicity of the FAdV strains responsible for the first reported case of IBH in South Africa. Polymerase chain reaction (PCR) amplification of the L1 loop region of the fowl adenovirus hexon gene using degenerate primer pair hexon A/B was used to identify the viruses that were isolated. Restriction fragment length polymorphism (RFLP) of the amplification products was used for the differentiation of 14 isolates of fowl adenovirus. Sequencing of the PCR products followed by amino acid comparison and phylogenetic analysis using the L1 loop region of the hexon protein was done to determine the identity of the isolates. Amino acid sequences of the hexon genes of all the South African isolates were compared with those of reference strains representing FAdV species. Amino acid comparison of 12 South Africa field isolates to FAdV reference strains revealed a high sequence identity $(>93.33 \%$ ) with reference strains T8-A and 764 . Two of the isolates had high sequence identity (93.40\%) with reference strains P7-A, C2B and SR48. Phylogenetic analysis of the L1 loop region of the hexon protein of all 14 South African isolates was consistent with their RFLP clusters. The mortality rates of embryos challenged with $10^{6}$ egg infective doses $\left(\right.$ EID $_{50}$ ) FAdV 2 were $80 \%$ - 87\% and mortality rates for embryos challenged with $10^{5.95}\left(\mathrm{EID}_{50}\right)$ FAdV 8 b were $65 \%-80 \%$.

\section{Introduction}

According to the International Committee on Taxonomy of Viruses (ICTV), host range specificity, genomic organisation, virion properties, structure, size and serological differences divide the large family of Adenoviridae into four distinct genera (Benkő et al. 2005). At present, these genera are Mastadenovirus (infecting mammals), Aviadenovirus (infecting birds), Atadenovirus (named after the bias of their genomes containing high $\mathrm{A}+\mathrm{T}$ content infecting a broad range of hosts including ruminants, as well as avian, reptilian and marsupial hosts), and Siadenovirus (infects birds and frogs). The fifth suggested genus is called Ichtadenovirus, isolated from fish (Benkó et al. 2005; Kovács et al. 2003).

The genus Aviadenovirus is further divided into eight species: Falcon adenovirus A, Fowl adenovirus (FAdV) A, Fowl adenovirus B, Fowl adenovirus C, Fowl adenovirus D, Fowl adenovirus E, Goose adenovirus $A$ and Turkey adenovirus $B$. Fowl adenovirus 1 (FAdV-1) belongs to species $\mathrm{A}$ and FAdV-5 to species B. FAdV-4 and FAdV-10 are grouped together in species C. Fowl adenovirus species D contains FAdV-2, FAdV-3, FAdV-9 and FAdV-11. FAdV-6, FAdV-7 and FAdV-8a and FAdV-8b are members of species E. Inconsistency of type numbering and selection of prototypes of FAdV-8 between different countries has led to recognition of two FAdV-8 subtypes ( $a$ and $b$ ) (Benkő et al. 2005).

Fowl adenoviruses (FAdVs) are common infectious agents of birds involved in a number of economically important diseases in poultry (McFerran \& Smyth 2000) and are implicated in inclusion body hepatitis (IBH) associated with mortalities in chickens from 2-6 weeks of age (Winterfield, Fadly \& Gallina 1973). Depending on the nature of the disease, the first sign of infection is a sudden increase in mortality of between $5 \%$ and $20 \%$. Diseases associated with FAdV commonly occur in the USA, Canada, UK, Australia, Europe, Ireland, India and Korea. Despite the worldwide distribution of FAdV-associated IBH, South Africa (SA) has until recently apparently been free of IBH. 
With respect to several characteristics of the virion (virus morphology, genome length and genome organisation), avian adenoviruses are heterogeneous. There seems to be no or little correlation between FAdV species and pathogenicity (Erney, Barr \& Fahey 1991). Serological methods to distinguish FAdV isolates are of little significance. Serum neutralisation cannot clearly differentiate between FAdV isolates (Benkő et al. 2005). Adenoviruses can be identified by electron microscopy, as their characteristic morphology of nonenveloped icosahedral particles $70 \mathrm{~nm}-100 \mathrm{~nm}$ in diameter can be easily recognised (Horne et al. 1959; McFerran 1998). Electron microscopy alone, however, cannot provide groupspecific information and subgroup-specific information.

Although more advanced molecular classification sytems, such as high-resolution melting-curve analysis of the FAdV hexon L1 gene region, have been proposed to classify FAdV more precisely, they cannot at this stage completely replace sequencing coupled with phylogenetic analysis (Marek et al. 2010). Aviadenovirus species designation therefore depends mainly on calculated phylogenetic distances of more than $5 \%-10 \%$ based on the protease pVIII hexon and DNA polymerase amino acid sequences, restriction fragment length polymorphism (RFLP) analysis, host range, pathogenicity, ability to recombine and cross-neutralisation (Benkő et al. 2005; McFerran 1998; McFerran \& Smyth 2000; Monreal, Dorn \& Kassim 1980).

Three different structural proteins (penton base, fibre and hexon) represent the type-specific and antigenic determinants of the adenovirus capsid (Horwitz 1996). The penton base contains only a few epitopes of minor importance in neutralisation and group-specificity (Valentine \& Pereira 1965). The hexon and fibre proteins are non-covalently linked to the penton base. Adenoviral fibres contain the adenovirus receptor (CAR) for attachment to host cells (Benkő et al. 2005) and some type-specific epitopes (Norrby 1969). The hexon protein is the major capsid protein and consists of conserved pedestal regions flanking variable loops (Raue \& Hess 1989). The L1 loops at the top of the hexon molecule form the outer surface of the virion and contain several important groupspecific and subgroup-specific epitopes (Norrby 1969) with high-sequence variability between the different FAdVs (Morães et al. 1998). Nucleic acid sequences of these typespecific epitopes have been used as phylogenetic markers to establish the relationship between European and American aviadenoviruses (Meulemans et al. 2004).

Phylogenetic analysis by amino acid alignment of the L1 hexon loops alone may place homonymous strains and nearly homonymous strains in different clusters, whilst RPFL analysis of such strains shows identical patterns (Meulemans et al. 2004). Polymerase chain reaction (PCR) amplification of the L1 loop region of the FAdV hexon gene with degenerate primer pair hexon A/and hexon B followed by RFLP of the gene products with a set of six restriction enzymes (BsiW1, Sty1, Mlu1, Sca1, Bgl1 and Asp1) is consistent with phylogenetic analysis and provides an attractive alternative to classic diagnostics and sequencing for detection and typing of new isolates of FAdV (Meulemans et al. 2004).
The present study was conducted to characterise and determine the pathogenicity of the recently isolated FAdV's associated with IBH in broiler chicks in SA.

\section{Materials and methods Origin of samples}

A recent outbreak of IBH caused significant production losses $(1 \%-5 \%)$ in broilers originating from young breeder flocks of an integrated broiler production enterprise in SA. The grandparent stock from this enterprise had been recently introduced into the country. Liver samples were collected during necropsy from broilers that showed increased mortality from 5-12 days of age with macroscopic lesions suggestive of IBH, including: hepatomegaly, hepatic necrosis with or without haemorrhage, an irregular surface and friable consistency of the liver.

\section{Virus isolation}

Liver samples, which were collected during necropsy from broilers, were inoculated intravascularly into the exposed blood vessels of embryonated chicken eggs. Embryonated eggs, which had been incubated at $37^{\circ} \mathrm{C}$ for $11-12$ days, were obtained from a specific pathogen free (SPF) White Leghorn flock (Avifarms [Pty] Ltd, PO Box 14167, Lyttelton 0140, SA). Eggs were candled and suitable blood vessels of the chorio-allantoic membrane were marked on the shells. Windows $(4 \mathrm{~mm} \times 8 \mathrm{~mm}$ ) were created in the shells to expose the selected chorio-allantoic blood vessels, according to established methods (Foster \& Luedke 1968). Liver samples collected during autopsy from broilers were diluted 1:100 and inoculated intravascularly into the exposed blood vessels with a $1 \mathrm{~mL}$ syringe (Terumo Medical Corporation, Japan) and a 27-gauge needle. The windows were sealed with a drop of non-toxic polyvinyl acetate dispersion (Ponal Wood glue). Eggs were incubated at $37^{\circ} \mathrm{C}$ for 7 days. Embryo mortalities within $48 \mathrm{~h}$ were regarded as nonspecific and the embryos discarded. A maximum of two passages per sample were performed.

\section{Pathogenicity determination}

Ten SPF embryos were challenged with a FAdV-8 and a FAdV-2 isolate from each genetic cluster. The challenge material was titrated in embryonated SPF eggs and standardised to $10^{6.00}$ egg infective dose (EID) ${ }_{50}$ for FAdV-2 and $10^{5.95} \mathrm{EID}_{50}$ for FAdV-8. The embryos were inoculated intravenously with $0.1 \mathrm{~mL}$ of diluted infectious cell culture fluids. Eggs were observed daily for 6 days and deaths were recorded. At 18 days of age all embryos that survived the challenge were culled. Liver tissues of embryos that died and others that survived the challenge but were culled were examined for gross and microscopic lesions.

\section{Polymerase chain reaction}

Total viral DNA was extracted for IBH using the High Pure Viral Nucleic Acid extraction kit (Rochè, Johannesburg, 
South Africa). Briefly, Aviadenovirus-infected chicken embryo liver tissue $(200 \mu \mathrm{L})$ with gross lesions associated with IBH was homogenised. Viral nucleic acids were purified from hepatocytes by incubation in Lysis/Binding Buffer in the presence of Proteinase K. Cell lysates containing the viral nucleic acids were mixed with a chaotropic salt and applied to the glass fiber fleece in a High Pure Spin Filter Tube (Rochè, Johannesburg, South Africa). Contaminating substances were removed from viral nucleic acids bound to the glass fleece with wash-and-spin steps and nucleic acids were eluted with $50 \mu \mathrm{L}$ water.

Degenerate primer pair hexon A/B described by Meulemans et al. (2001) and synthesised by Inqaba Biotech (Pty) Ltd (Muckleneuk, Pretoria) was used to amplify position 1441041 of the L1 encoding region of the hexon protein. The PCR amplification conditions were as described by Meulemans et al. (2001). The $50 \mu \mathrm{L}$ PCR reaction contained $2 \mu \mathrm{L}$ viral DNA, 125 pmoles of each primer, 2.5 U Pyrobest polymerase (Takara, Bio Inc., Shiga, Japan), $5 \mu \mathrm{L} 10 \mathrm{x}$ supplied Pyrobest buffer and $7.5 \mathrm{mM}$ dNTPs. Amplification products were separated on $2 \%$ agarose gels stained with cybergold (Invitrogen, Carlsbad, California, USA) and visualised using a dark reader. The middle range Fastruler (Fermentas, Thermo Fisher Scientific Inc., Massachusetts, USA) and 50 bp Generuler (Fermentas, Thermo Fisher Scientific Inc.) or $50 \mathrm{bp}$ ladder (Promega Corporation Wisconsin, USA) were loaded on the $2 \%$ agarose gel together with the amplification products. PCR products of $~ 900$ base pairs were excised from the $2 \%$ agarose gel and purified using the Zymoclean ${ }^{\mathrm{TM}}$ Gel DNA Recovery Kit (ZYMO Research Corp., California, USA). Infectious bursal disease (IBD) reverse transcription PCR was performed on a FAdV-infected chicken embryo liver, spleen and bursa tissue suspected to also be infected with IBD virus.

\section{Nucleotide sequencing of the L1 loop of the hexon protein}

Polymerase chain reaction amplification products purified from the $2 \%$ agarose gel were ligated into sequencing vector pJet 1.2 using the CloneJET PCR Cloning Kit from Fermentas (Thermo Fisher Scientific Inc., Massachusetts, USA), following the cloning protocol for blunt ends. Sequencing was performed by Inqaba Biotechnical Industries (Pty) Ltd, with the pJet 1.2 forward and reverse-sequencing primers supplied with the CloneJET PCR Cloning Kit from Fermentas. All bla ${ }_{\text {pjet }}$-specific PCR amplicons were sequenced with the pJet forward primer using an ABI 3130XL genetic analyser (Applied Biosystems, California, USA), incorporating the ABI Big Dye Terminator Cycle Sequencing Kit version

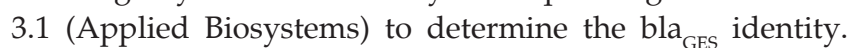
Sequencing was performed by Inqaba Biotech (Pty) Ltd. Electropherograms of the sequences generated were inspected with FinchTV (Geospiza Inc., Washington DC, USA). Sequences were assembled and conflicts resolved using CLC Bio Version 5 software (CLC bio, Finlandsgade, Denmark). Sequences were copied and aligned using the Basic Local Alignment Search Tool (BLAST) with the blasn algorithm for highly similar sequences (Altschul et al. 1997).
Sequences were deposited in Genbank under accession numbers HQ117898 to HQ117911.

\section{Restriction enzyme analysis of the L1 loop of the hexon protein}

Gel purified PCR products were used for restriction enzyme analysis. Restriction enzymes BsiWI, Sty1, Mlu1, Sca1, Asp1 and $B g l 1$ were used to determine the restriction patterns of the virus hexon amplification products. Restriction enzyme digests were carried out in a total volume of $20 \mu \mathrm{L}$ containing $5 \mu \mathrm{L}$ PCR product and $1 \mathrm{U}$ restriction enzyme. The reactions were carried out according to the instructions of the supplier (Fermentas, Takara). Cleavage products were separated on a $3 \%$ agarose gel stained with cybergold (Invitrogen) for $40 \mathrm{~min}$ at $125 \mathrm{~mA}$ and visualised using a dark reader. Restriction enzyme patterns obtained from the gels were documented using a digital camera. To confirm the results obtained by restriction enzyme digests with BsiW1, Sty1, Mlu1, Sca1, Asp1 and Bgl1, the theoretical restriction enzyme profiles L1 hexon loop of all 14 SA isolates were calculated on the nucleotide sequences obtained, using the restriction enzyme search function from the CLC Bio Version 5 software package.

\section{Pairwise comparison between L1 loop amino acid sequences}

Nucleotide sequences were translated to amino acid sequences using CLC Bio Version 5 software (CLC bio, Finlandsgade, Denmark). Pairwise identity of translated amino acid sequences was calculated using the online MAFFT progressive algorithm software (Katoh et al. 2002). Pairwise identity of all SA isolates was determined by alignment to the reference strains listed in Table 1.

\section{Phylogenetic analysis of the loop 1 peptide sequences}

Fowl adenovirus hexon sequences (Table 1) were obtained from the public databases by BlastP search at NCBI (http:/ / www.ncbi.nlm-nih.gov/BLAST/). The sequence from the hexon of strain CELO (accession number AAL 13217) was used as search term. Thirty three non-redundant sequences, which included a segment of 297 amino acids within the L1 loop of the hexon protein, were recovered. These sequences corresponded to residues 49-346 of the hexon protein of the reference strain CELO (Chiocca et al. 1996).

The Neighbour-Joining algorithm was used to estimate evolutionary history (Saitou \& Nei 1987) and 1000 replicates used in the bootstrap test. The percentage of replicate trees that clustered together are indicated by the values next to the branches (Felsenstein 1985). The tree was drawn to scale with branch lengths in the same as the evolutionary distances used to infer the phylogenetic tree. The evolutionary distances were calculated with the Dayhoff matrix-based method (Schwarz \& Dayhoff 1979), which reflects the number of amino acid substitutions per site. Alignment gaps and missing data were eliminated only in pairwise sequence comparisons (pairwise deletion option). There were a total of 297 positions in the final dataset. Phylogenetic analyses were conducted in MEGA4 (Tamura et al. 2007). 


\section{Results}

\section{Pathogenicity}

The mortality rates of embryos were $80 \%-87 \%$ and $65 \%-80 \%$ for FAdV-2 and FAdV-8, respectively. Livers of embryos that survived challenge were enlarged and mottled with small, alternating, dull grey, yellow-brown and reddish-brown foci (hepatic necrosis), whilst in others the liver showed a diffuse greenish discolouration (bile stasis) with scattered, irregular, grey foci that coalesced in some areas (Figure 1a and 1b). In

TABLE 1: Fowl adenovirus reference strains. Fowl adenovirus strains used for phylogenetic analysis.

\begin{tabular}{|c|c|c|c|c|}
\hline \multirow[t]{2}{*}{ Fowl adenovirus species } & \multicolumn{4}{|c|}{$\begin{array}{l}\text { Serotypes or strains, the isolates that represented them and the accession numbers of the hexon sequences used for the } \\
\text { phylogenetic analysis }\end{array}$} \\
\hline & Type & ICTV & USA & EUROPE \\
\hline \multirow[t]{4}{*}{ Fowl adenovirus A } & FAdV1 & CELO (AAL13217) & QBV & CELO (AAL13217) \\
\hline & & CELO (NP_043886) $\dagger$ & Indiana CT3 & - \\
\hline & & 112 & QT & - \\
\hline & & Phelps (AAL13217) & - & - \\
\hline \multirow[t]{5}{*}{ Fowl adenovirus B } & FAdV5 & 340 (AAN77078) & - & - \\
\hline & FAdV3 & - & 340 (AAN77076) & - \\
\hline & & - & $\mathrm{M} 2$ & - \\
\hline & & - & IBH-2A (AAL13219) & - \\
\hline & & - & Tipton & - \\
\hline \multirow[t]{8}{*}{ Fowl adenovirus C } & FAdV4 & KR95 (CAD2447) & 506-1 (AAN77076) & Ganesh (AAF25605) India \\
\hline & & $\mathrm{J} 2$ & HR-5 & Sheppard (AAA91647) India \\
\hline & & KR5 (AAN77077) & - & Barua (CAD86791) Russia \\
\hline & & J2A (AAL13220) & - & Indian (CAD30847) India \\
\hline & FAdV10 & CFA 20 & C2B (AAL13226) & - \\
\hline & & C2B (AAL13226) & - & - \\
\hline & & M11 & - & - \\
\hline & FAdV11 & - & - & C2B (AAN77085) \\
\hline \multirow[t]{12}{*}{ Fowl adenovirus D } & FAdV2 & P7-A (AAL13218) & GAL-1A & GAL-1 \\
\hline & & 685 (AAN77073) & P7 & - \\
\hline & & - & $\mathrm{Z7}$ & - \\
\hline & & - & SR-48 (AAN77072) & - \\
\hline & FAdV3 & 75 (AAN77075) & - & - \\
\hline & & SR49 (AAN77074) & - & - \\
\hline & FAdV5 & TR22 (AAN77079) & - & CR119 (AAN77080) \\
\hline & FAdV6 & CR119 (AAN77080) & - & 75-1A-1 (AAL13224) \\
\hline & FAdV9 & A2-A (AAL13221) & $\mathrm{A} 2$ & - \\
\hline & FAdV10 & - & - & A-2A (NP_050287) $\dagger$ \\
\hline & FAdV11 & 380 (AAL13228) & - & - \\
\hline & FAdV12 & - & - & 380 \\
\hline \multirow[t]{21}{*}{ Fowl adenovirus $\mathrm{E}$} & FAdV5 & - & 58-1 (AAN77083) & - \\
\hline & & - & $\mathrm{T}-8$ & - \\
\hline & & - & TR-59 & - \\
\hline & & - & U-6 & - \\
\hline & & - & $Q-1 A$ & - \\
\hline & FAdV7 & YR36 (AAN77081) & 764 (AAN77084) & $x-11$ \\
\hline & & $x-11$ & B3A (AAL13225) & - \\
\hline & & x11A (AAL13227) & - & - \\
\hline & & $\mathrm{x} 11 \mathrm{a}$ like virus & - & - \\
\hline & FAdV8 & - & - & TR59 (AAN77082) \\
\hline & & - & - & A2-A (AAL13221) \\
\hline & FAdV8a & TR59 (AAN77082) & - & - \\
\hline & & $\mathrm{T}-8$ & - & - \\
\hline & & CFA40 & - & - \\
\hline & & T8-A (AAL13222) & - & - \\
\hline & FAdV8b & Stanford5 & - & - \\
\hline & & 764 (AAN77084) & - & - \\
\hline & & B3 & - & - \\
\hline & FAdV8a/b & Ontario (ICTV) & - & - \\
\hline & FAdV9 & - & - & 764 (AAN 77084) \\
\hline & FAdV10 & - & $x-11$ & - \\
\hline
\end{tabular}

Source: Adapted from Ekanayake, S., 2009, 'Inclusion body hepatitis as a primary disease in commercial broiler chickens', MSc dissertation, Department of Veterinary Pathology, University of Saskatchewan, viewed 18 November 2013, from http://ecommons. usask.ca/bitstream/handle/10388/etd-01062010-145947/Samantha. EkanayakeThesisMSc.pdf?sequence=1

FAdV, Fowl adenovirus; ICTV, International Committee on Taxonomy of Viruses Fowl adenovirus strains; USA, United States of America Fowl adenovirus strains; EUROPE, European strains. $\dagger$, complete genome sequences. 
dead embryos, the autolysed livers were small and yellow with a barely visible faint, white stippling. Lesions in the spleen included prominent splenomegaly and indistinct, grey stippling. The mesonephros and metanephros of some affected embryos were swollen and showed a blackish discolouration due to severe congestion and haemorrhage. In some embryos, irregular ecchymoses occurred on the serosal surface of the gizzard and a whitish, opaque effusion clouded the peritoneal cavity (most likely visceral gout) of a small proportion of affected embryos.

\section{Polymerase chain reaction amplification and restriction fragment length polymorphism analysis}

Fourteen of the fifteen fresh liver samples from chicken embryos inoculated with FAdV field isolates that displayed clinical signs of adenovirus were positive for FAdV, with PCR. Isolate SA69-08 tested negative on FAdV PCR but was confirmed to be positive for IBD. Isolate SA82-08 tested positive on FAdV and IBD PCR. Isolates identified as FAdV either by gross pathology, histopathology and isolation were confirmed positive by PCR for FAdV (Table 2). Their identities were confirmed by nucleotide sequence comparison to the ICTV reference strains listed in Table 1.

Polemerase chain reaction amplification products of the 14 fowl adenovirus isolates were purified from the agarose. The amplification products were used for restriction enzyme analysis to determine their restriction enzyme type. Endonuclease digestion, using restriction enzymes BsiW1, Sty1, Mlu1, Sca1, Asp1 and Bgl1 could differentiate between SA isolates (SA38C-08, SA38D-08, SA56-08, SA59-08, SA6208, SA63-08, SA64-08, SA65-08, SA78-08, SA82-08, SA83-08, SA84-08, SA55-08 and SA58-08). Restriction enzyme patterns obtained by digestion with the six restriction enzymes placed isolates SA38C-08, SA38D-08, SA56-08, SA59-08, SA62-08,
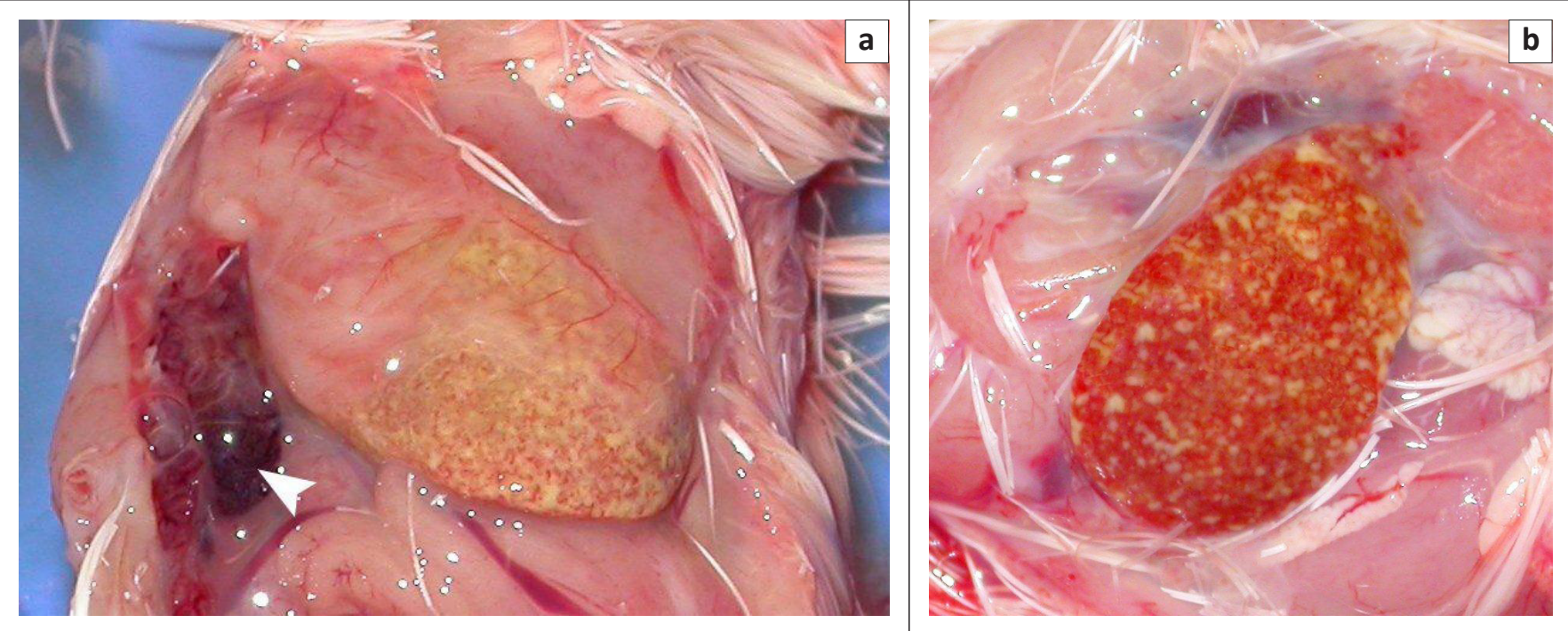

Note: (a) prominent grey and orange-brown mottling of a moderately enlarged liver. The kidneys (white arrow head) show moderate swelling and congestion; (b) note the diffuse reddish-brown and grey mottling of the moderately enlarged liver.

FIGURE 1: (a) Organs from an affected embryo seven-days post inoculation, (b) organs from an embryo that died 5-days post inoculation.

TABLE 2: Summarised results of virus isolation.

\begin{tabular}{|c|c|c|c|c|c|}
\hline Sample ID & $\begin{array}{l}\text { Gross } \\
\text { pathology }\end{array}$ & Histopathology & Virus isolation & $\begin{array}{l}\text { PCR } \\
\text { confirmation }\end{array}$ & $\begin{array}{l}\text { Nucleotide } \\
\text { sequence } \\
\text { homology }\end{array}$ \\
\hline SA38C-08 & $(+)$ & $(+)$ & $(+)$ & $\operatorname{AAV}(+)$ & FAdV-8b \\
\hline SA38D-08 & $(+)$ & $(+)$ & $(+)$ & $\operatorname{AAV}(+)$ & FAdV-8b \\
\hline SA55-08 & $(+)$ & $(+)$ & $(+)$ & $\operatorname{AAV}(+)$ & FAdV-2 \\
\hline SA56-08 & $(-)$ & $(+)$ & $(+)$ & $\operatorname{AAV}(+)$ & FAdV-8b \\
\hline SA58-08 & $(+)$ & $(+)$ & $(+)$ & $\operatorname{AAV}(+)$ & FAdV-2 \\
\hline SA62-08 & $(+)$ & $(+)$ & $(+)$ & $\operatorname{AAV}(+)$ & FAdV-8b \\
\hline SA63-08 & $(+)$ & $(+)$ & $(+)$ & $\operatorname{AAV}(+)$ & FAdV-8b \\
\hline SA64-08 & $(+)$ & $(+)$ & $(+)$ & $\operatorname{AAV}(+)$ & FAdV-8b \\
\hline SA65-08 & $(+)$ & $(+)$ & $(+)$ & $\operatorname{AAV}(+)$ & FAdV-8b \\
\hline SA69-08 & $(-)$ & $(-)-$ & $(+)$ & $\operatorname{IBD}(+)$ & IBD D78 \\
\hline SA78-08 & $(+)$ & $(+)$ & $(+)$ & $\operatorname{AAV}(+)$ & FAdV-8b \\
\hline SA82-08 & $(+)$ & $(-)$ & $(+)$ & $\mathrm{AAV} ; \mathrm{IBD}(+)$ & FAdV-8b; IBD $\dagger$ \\
\hline SA84-08 & $(+)$ & $(+)$ & $(+)$ & $\operatorname{AAV}(+)$ & FAdV-8b \\
\hline
\end{tabular}

PCR, Poymerase chain reaction; AAV, Avian adenovirus; IBD, infectious bursal disease; FAdV, Fowl adenovirus.

$\dagger$, positive for infectious bursal disease; $(+)$, positive; $(-)$, negative. 
SA63-08, SA64-08, SA65-08, SA78-08, SA82-08, SA83-08 and SA84-08 in the same restriction enzyme cluster. Restriction enzyme patterns for isolates SA55-08 and SA58-08, using the six restriction enzymes, were different from those of the other SA isolates and placed them into a different RFLP cluster. Restriction enzymes Asp1 and Bgl1 did not cut any of the amplification products of the SA isolates. Results of this analysis for enzymes BsiW1, Sty1, Mlu1 and Sca1 are presented in Figures $2 \mathrm{a}-\mathrm{d}$. Calculation of the theoretical restriction enzyme profiles using the nucleic acid sequence of the L1 loop of the hexon protein of all isolates confirmed the results obtained with the RFLP analysis done with the six restriction enzymes (BsiWI, Sty1, Mlu1, Sca1, Asp1 and Bgl1). The calculated sizes of the RE fragments are summarised in Table 3.

\section{Pairwise comparisons between L1 loop amino acid sequences}

Sequencing of the PCR amplification indicated that 12 of the 14 virus isolates were closely related and shared DNA sequence identity (99\%) with reference strain T8-A (Genbank: AF339919). The other two were closely related to each other and shared DNA sequence identity (99\%) with ATCC reference strain P7-A (Genbank: AF339915).

Isolates SA38C-08, SA82-08 and SA84-08 had 99.00\% amino acid sequence identity to reference strains T8-A and 764 and showed $93.00 \%$ identity to reference strains YR36 and X11. Amino acid sequence identity of isolates SA38C-08, SA82-08 and SA84-08 to B-3A, X11A and CR119 were 93.67\%. Isolates SA 38C-08, SA82-08 and SA84-08 shared a sequence identity of $99.33 \%$ with each other and were closely related to the other SA isolates (99.67\%). Isolates SA55-08 and SA 58-08 had amino acid sequence identities of $79.93 \%$ and $83.33 \%$ with SA38C-08, SA82-08 and SA84-08.

The amino acid sequences of SA isolates SA38D-08, SA56-08, SA59-08, SA62-08, SA63-08, SA64-08, SA65-08 and SA78-08 were $100 \%$ identical. All these isolates had the same sequence identity to reference strains T8-A and 764 (99.33\%) and to YR36 and X11, 93.33\% and 98.67\%, respectively. They also shared amino acid sequence identity of $89.67 \%$ with reference strain CR119.

Fowl adenovirus isolates SA55-08 and SA58-08 were clearly distinct from the other SA isolates that share high amino acid sequence identity with the FAdV species E, as they only shared sequence identity of between $79.93 \%$ and $83.33 \%$ with the other SA isolates and reference strains T8-A and 764 . Isolate SA55-08 had the highest sequence identity to FAdV reference strain P7-A (96.01\%), the same sequence identity to reference strains C2B and SR48 (94.35\%) and 94.02\% and $91.69 \%$ to reference strains 380 and 685 , respectively. South African isolates SA58-08 shared $98.66 \%$ amino acid sequence identity with reference strain P7-A, 96.99\% amino acid sequence identity with C2B and SR48 and $96.66 \%$ with reference strain 380 . Isolate SA 58-08 shared $94.31 \%$ identity with reference strain 685 . The amino acid sequence of isolate SA55-08 shared 94.39\% identity with SA58-08.

\section{Phylogenetic analysis}

Results of the phylogenetic analysis by the distance method are shown in Figure 3. Phylogenetic characterisation of the FAdVs place the SA isolates into two clusters. Isolates SA38C-08, SA38D-08, SA56-08, SA59-08, SA62-08, SA63-08,

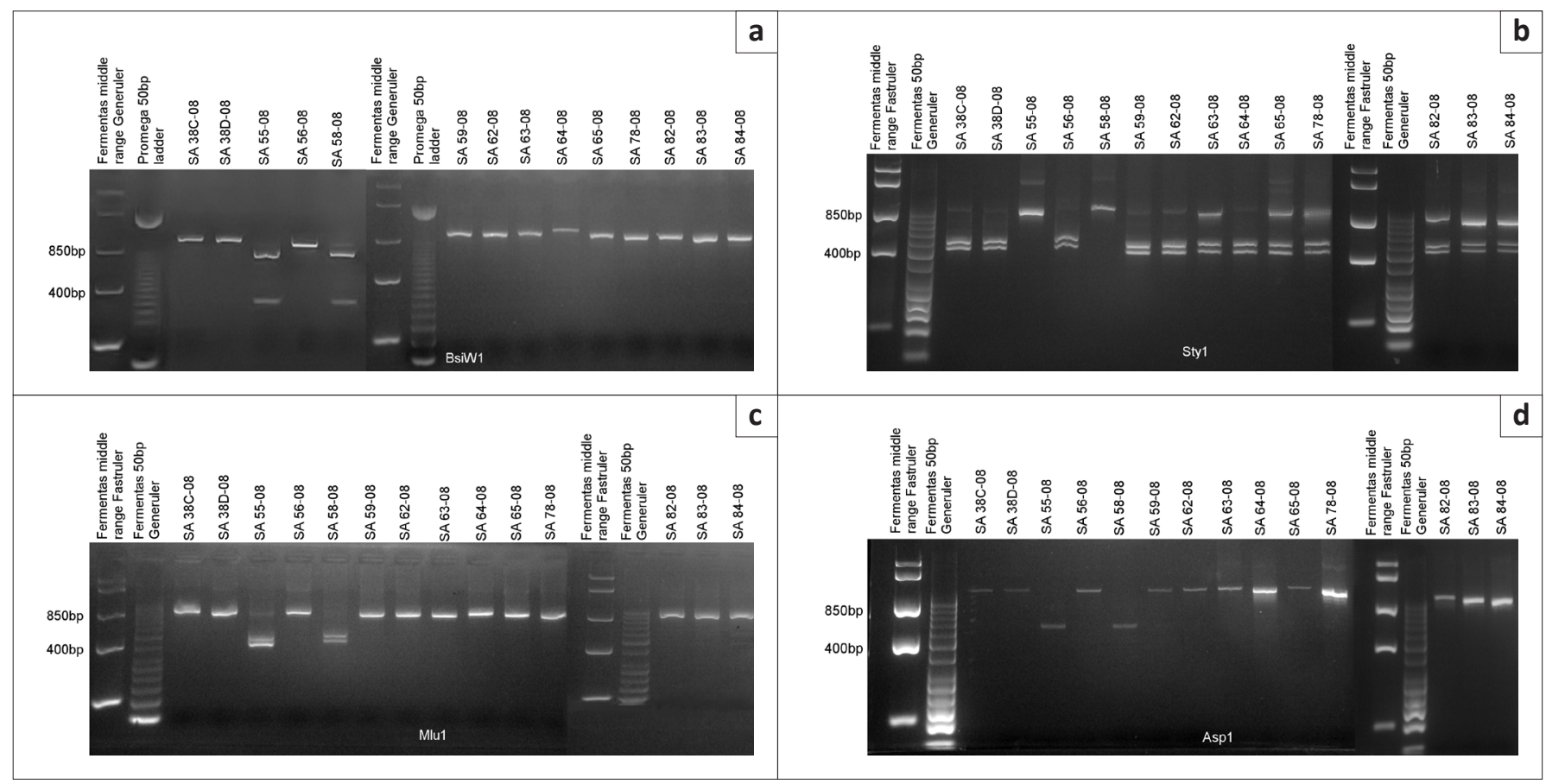

FIGURE 2: (a) BsiW1 restriction enzyme digests of the L1 loop region of the polymerase chain reaction product generated with the hexon $1 /$ hexon 2 primer set, (b) Sty1 restriction enzyme digests of the L1 loop region of the polymerase chain reaction product generated with the hexon 1 /hexon 2 primer set, (c) Mlu1 restriction enzyme digests of the L1 loop region of the polymerase chain reaction product generated with the hexon 1/hexon 2 primer set and (d) Asp 1 restriction enzyme digests of the L1 loop region of the polymerase chain reaction product generated with the hexon 1 /hexon 2 primer set. 

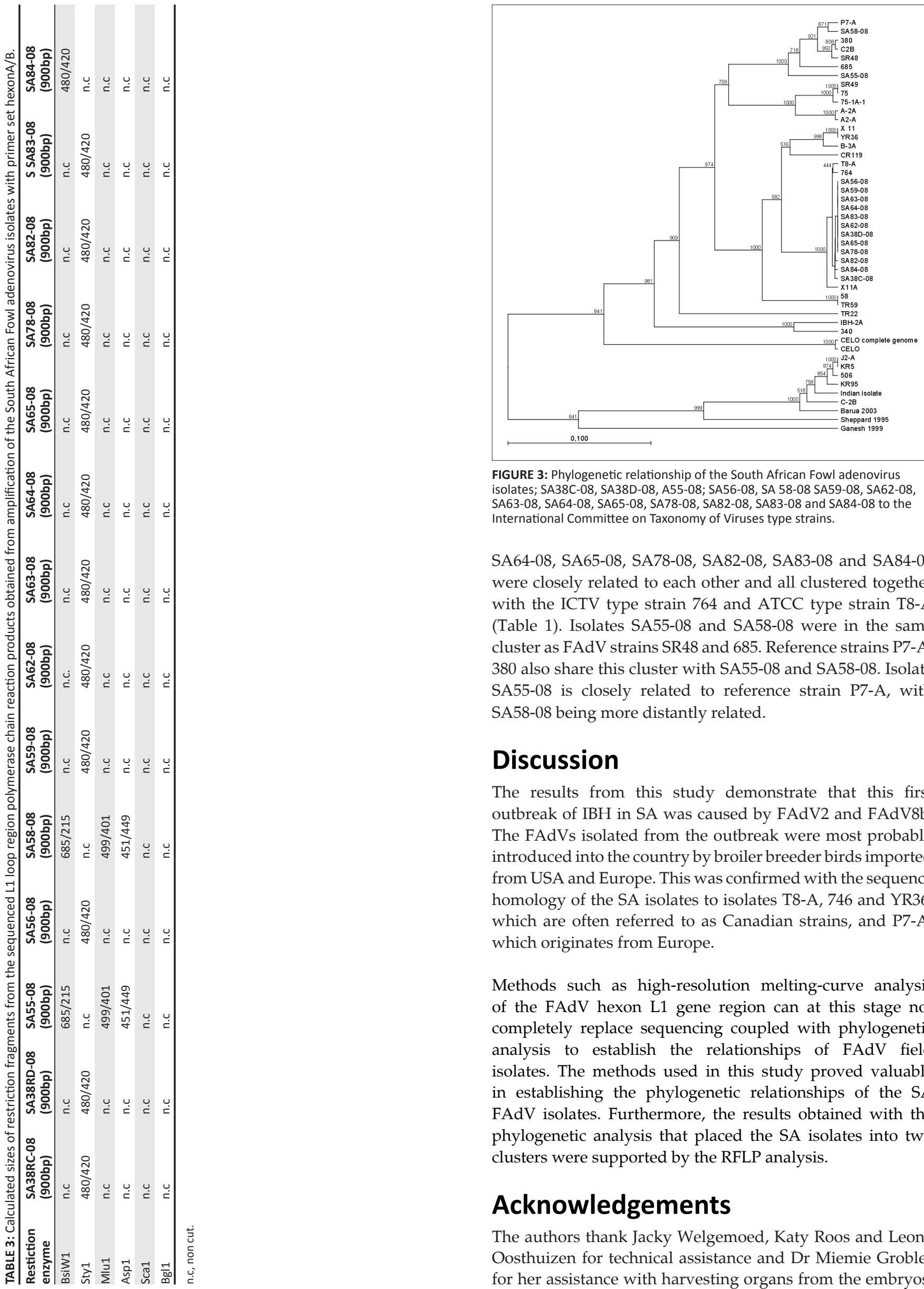

FIGURE 3: Phylogenetic relationship of the South African Fowl adenovirus isolates; SA38C-08, SA38D-08, A55-08; SA56-08, SA 58-08 SA59-08, SA62-08, SA63-08, SA64-08, SA65-08, SA78-08, SA82-08, SA83-08 and SA84-08 to the International Committee on Taxonomy of Viruses type strains.

SA64-08, SA65-08, SA78-08, SA82-08, SA83-08 and SA84-08 were closely related to each other and all clustered together with the ICTV type strain 764 and ATCC type strain T8-A (Table 1). Isolates SA55-08 and SA58-08 were in the same cluster as FAdV strains SR48 and 685. Reference strains P7-A, 380 also share this cluster with SA55-08 and SA58-08. Isolate SA55-08 is closely related to reference strain P7-A, with SA58-08 being more distantly related.

\section{Discussion}

The results from this study demonstrate that this first outbreak of IBH in SA was caused by FAdV2 and FAdV8b. The FAdVs isolated from the outbreak were most probably introduced into the country by broiler breeder birds imported from USA and Europe. This was confirmed with the sequence homology of the SA isolates to isolates T8-A, 746 and YR36, which are often referred to as Canadian strains, and P7-A, which originates from Europe.

Methods such as high-resolution melting-curve analysis of the FAdV hexon L1 gene region can at this stage not completely replace sequencing coupled with phylogenetic analysis to establish the relationships of FAdV field isolates. The methods used in this study proved valuable in establishing the phylogenetic relationships of the SA FAdV isolates. Furthermore, the results obtained with the phylogenetic analysis that placed the SA isolates into two clusters were supported by the RFLP analysis.

\section{Acknowledgements}

The authors thank Jacky Welgemoed, Katy Roos and Leona Oosthuizen for technical assistance and Dr Miemie Grobler for her assistance with harvesting organs from the embryos. 
Thank you to Dr Carine Pienaar and Prof. Estelle Venter for assistance with editing the manuscript.

\section{Competing interests}

The authors declare that they have no financial or personal relationship(s) that may have inappropriately influenced them in writing this article.

\section{Authors' contributions}

H.W.J. (Deltamune) conceptualised, performed the work, interpreted the results and wrote the article. L.H.M. (Deltamune) provided advice and guidance for the isolation and identification of viruses in this study and contributed to the writing of the article. H.A. (Avimune) identified the disease in the field and collected the samples that were used during the study. E.H.V. (University of Pretoria) assisted with advise and guidance and contributed to the editing of the manuscript.

\section{References}

Altschul, S.F., Madden, T.L., Schäffer, A.A., Zhang, J., Zhang, Z. \& Miller, W., 1997 'Gapped BLAST and PSI-BLAST: A new generation of protein database search programs', Nucleic Acids Research 25(17), 3389-3402, viewed 11 November 2013, from http://www.ncbi.nlm.nih.gov/pubmed/9254694

Benkő, M., Harrach, B., Russel, W.C., Adair, B.M., Ádám, É. \& De Jong, J.C., 2005 'Family Adenoviridae', in C.M. Fauquet, M.A. Mayo, J. Maniloff, U. Desselberger \& L.A. Ball (eds.), Virus Taxonomy, classification and nomenclature of viruses: VIIIth Report of the International Committee on Taxonomy of Viruses, pp. 213-226, Elsevier/Academic Press, London.

Chiocca, S., Kurzbauer, R., Schaffner, G., Baker, A., Mautner, V. \& Cotton, M., 1996 'The complete DNA sequence and genomic organization of the avian adenovirus CELO', Journal of Virology 70(5), 2939-2949, viewed 11 November 2013, from http://0-jvi.asm.org.innopac.up.ac.za/content/70/5/2939.full.pdf+html

Ekanayake, S., 2009, 'Inclusion body hepatitis as a primary disease in commercial broiler chickens', MSc dissertation, Department of Veterinary Pathology, University of Saskatchewan, viewed 18 November 2013, from http://ecommons. usask.ca/bitstream/handle/10388/etd-01062010-145947/Samantha. EkanayakeThesisMSc.pdf?sequence $=1$

Erney, K.M., Barr, D.A. \& Fahey, K.J., 1991, 'Molecular characterization of highly virulent fowl adenoviruses associated with outbreaks of inclusion body hepatitis' Avian Pathology 20(4), 597-606, viewed 11 November 2013, from http://0-www. tandfonline.com.innopac.up.ac.za/doi/pdf/10.1080/03079459108418799

Felsenstein, J., 1985, 'Confidence limits on phylogenies: An approach using the bootstrap', Evolution 39(4), 783-791, viewed 11 November 2013, from http://0www.jstor.org.innopac.up.ac.za/stable/pdfplus/2408678.pdf?acceptTC=true\&ac ceptTC=true\&jpdConfirm=true

Foster, N.M. \& Luedke, A.J., 1968, 'Direct assay for bluetongue virus by intravascular inoculation of embryonated chicken eggs', American Journal of Veterinary Research 29(3) 749-775.

Horne, R.W., Bonner, S., Waterson, A.P. \& Wildy, P., 1959, 'The icosahedral form of an adenovirus', Journal of Molecular Biology 1(1), 84-86, viewed 23 November 2013 from http://0-ac.els-cdn.com.innopac.up.ac.za/S0022283659800115/1 s2.0-S0022283659800115-main.pdf? tid=dd9f7d2e-5510-11e3-9f79-00000aacb 35d\&acdnat=1385301847_171e9b97730f76d79f6f10bb5e8e6b06

Horwitz, M.S., 1996, 'Adenovirus', in B.N. Fields, D.M. Knipe \& M.D. Howley (eds.), Virology, 3rd edn., pp. 2149-2171, Lippincott Publishers, Philadelphia.
Katoh, K., Misawa, K., Kuma, K. \& Miyata, T., 2002, 'MAFFT: A novel method for rapid multiple sequence alignment based on fast Fourier transformation', Nucleic Acids Research 30(14), 3059-3066, viewed 23 November 2013, from http://0-nar. oxfordjournals.org.innopac.up.ac.za/content/30/14/3059.full.pdf+html

Kovács, G.M., LaPatra, S.E., D'Halluin, J.C. \& Benkő, M., 2003, 'Phylogenetic Analysis of the hexon and protease genes of a fish adenovirus isolated from white sturgeon (Acipenser transmontanus) supports the proposal for a new adenovirus genus', Virus Research 98(1), 27-34, viewed 18 November 2013, from http://0-ac.els-cdn. Virus Research 98(1), 27-34, viewed 18 November 2013, from http://0-ac.els-cdn
com.innopac.up.ac.za/S0168170203002508/1-s2.0-S0168170203002508-main pdf? tid=d9c1fafe-5be8-11e3-ae2d-00000aacb35f\&acdnat=1386054319_c315d pdf? ${ }^{\text {tid }}=$ d9c1 2 fafe-5be8-11e3-ae2d

Marek, A., Günes, A., Schulz, E. \& Hess, M., 2010, 'Classification of fowl adenoviruses by use of phylogenetic analysis and high-resolution melting-curve analysis of the hexon L1 gene region', Journal of Virological Methods 170(1-2), 147-154 viewed 23 November 2013 from http://0-ac.els-cdn com innol, $147-154$ viewed 23 November 2013, from http://0-ac.els-cdn.com.innopac.up.ac.za/ S0166093410003435/1-s2.0-S0166093410003435-main.pdf? -tid=6f06f5a6$5513-11$ e3-a9d

McFerran, J.B., 1998, 'Adenoviruses', in D.E. Swayne, R. John, M.W. Jackwood, J.E. Pearson \& W.M. Reed (eds.), A laboratory manual for the isolation and identification of avian pathogens, pp. 223-234, Kennett Square, PA, University of Pennsylvania, Pennsylvania.

McFerran, J.B. \& Smyth, J.A., 2000, 'Avian adenoviruses', Revue scientifique et technique, Office international des Épizooties 19(2) 589-601.

Meulemans, G., Boschmans, M., Van den Berg, T.P. \& Decaesstecker, M., 2001, 'Polymerase chain reaction combined with restriction enzyme analysis for detection and differentiation of fowl adenoviruses', Avian Pathology 30(6) 655660, viewed 23 November 2013, from http://0-web.ebscohost.com.innopac. up.ac.za/ehost/pdfviewer/pdfviewer?vid=3\&sid=276f47fa-c98d-4c0f-ac8a-1bd5 $8 \mathrm{e} 2 \mathrm{f} 6111 \% 40$ sessionmgr115\&hid=128

Meulemans, G., Couvreur, B., Decaesstecker, M., Boschmans, M. \& Van den Berg, T.P., 2004, 'Phylogenetic analysis of fowl adenoviruses', Avian Pathology 33(2), 164170, viewed 23 November 2013, from http://0-www.tandfonline.com.innopac. up.ac.za/doi/pdf/10.1080/03079450310001652086

Monreal, G., Dorn, R. \& Kassim, M., 1980, 'Detection of neutralizing antibodies against avian adenovirus in a microtitre cell-culture system', Berliner Münchner against avian adenovirus in a microtitre cell-
Tierärztliche Wochenschrift 93(7), 125-128.

Morães, M.T.B., Da Silva, M., Leite, J.P.G. \& Nascimento, J.P., 1998, 'Genetic and antigenic analysis of adenovirus type 3 strains showing intermediate behavior in standard seroneutralization test', Memórias do Instituto Oswaldo Cruz 93(2), 231-235, viewed 23 November 2013, from http://www.scielo.br/pdf/mioc/ v93n2/3420.pdf

Norrby, E., 1969, 'Comparative studies on the soluble components of adenovirus types 9 and 15 and the intermediate strain 9-15,' Journal of Virology 2(10), 12001210 , viewed 23 November 2013, from http://0-jvi.asm.org.innopac.up.ac.za/ content/2/10/1200.full.pdf+html

Raue, P. \& Hess, M., 1989, 'Hexon based PCRs combined with restriction enzyme analysis for rapid detection and differentiation of fowl adenovirus and egg drop syndrome virus', Journal of Virological Methods 73(2), 211-217, viewed 23 November 2013 from http://0-ac.els-cdn.com.innopac.up.ac.za/S0166093498000652/1-s2.0 S0166093498000652-main.pdf? tid=98b13630-551f-11e3-bb1c-00000aab0f27\& acdnat=1385308174_9eb3ec576̄b93a9b82fee08de3a66ba7a

Saitou, N. \& Nei, M., 1987, 'The neighbor-joining method: A new method for reconstructing phylogenetic trees', Molecular Biology and Evolution 4(4), 406425, viewed 23 November 2013, from http://0-mbe.oxfordjournals.org.innopac. up.ac.za/content/4/4/406.full.pdf+htm

Schwarz, R. \& Dayhoff, M., 1979, 'Matrices for detecting distant relationships', in M. Dayhoff, (ed.), Atlas of protein sequences, pp. 353-358, National Biomedical Research Foundation, Georgetown University, Washington DC.

Tamura, K., Dudley, J., Nei, M. \& Kumar, S., 2007, 'MEGA4: Molecular Evolutionary Genetics Analysis (MEGA) software version 4.0', Molecular Biology and Evolution 24(8), 1596-1599, viewed 23 November 2013, from http://0-mbe.oxfordjournals. org.innopac.up.ac.za/content/24/8/1596.full.pdf+html

Valentine, R.G. \& Pereira, H.G., 1965, 'Antigens and structure of the adenovirus', Journal of Molecular Biology 13(1), 13-20, viewed 23 November 2013, from hournal of Molecular Biology 13(1), 13-20, viewed 23 November 2013, from http://0-ac.els-cdn.com.innopac.up.ac.za/S0022283665800766/1-s2.0b\&acdnat $=1385382904 \quad 8 \mathrm{a} 4 \mathrm{a} a \mathrm{6a} 04 \mathrm{4}$ bfb3a9765220caffa30d60a3. http://dx.doi.

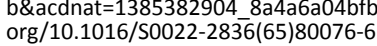

Winterfield, R.W., Fadly, A.M. \& Gallina, A.M., 1973, 'Adenovirus infection and disease. Some characteristics of an isolate from chickens in India', Avian Diseases 17(2), Some characteristics of an isolate from chickens in India', Avian Diseases $17(2)$,
$334-342$, viewed 23 November 2013, from http://0-www.jstor.org.innopac.up.ac. 334-342, viewed 23 Novemt
za/stable/view/1589217 